\title{
Evaluation of gas pipeline induced voltage associated with parallel transmission line
}

\begin{abstract}
The voltage will be induced in the pipeline due to an electromagnetic field effect generated by the parallel transmission tower. This will bring up the negative effect to the pipeline, transmission tower and people get contracts with the pipeline. A study has been carried out to determine the electromagnetic field (EMF) distribution of parallel transmission line and pipeline due to AC currents and lightning faults. Only a few of previous study focus on lightning faults. The lightning faults data for transmission line and pipeline still insufficient nowadays. A mitigation system has been developed to minimize the induced voltage on pipeline due to the electromagnetic field of energized transmission line. ANSYS Maxwell software is used for modeling. The range of induced voltage obtained is within the acceptable level of the IEEE 80-2000 standards.
\end{abstract}

Keyword: Eletromagnetic Field Distribution (EMF); Induced voltage 